\title{
A CHARACTERIZATION OF GROMOV HYPERBOLICITY OF SURFACES WITH VARIABLE NEGATIVE CURVATURE
}

\author{
ANA PORTILLA ${ }^{(1)}$ AND EVA TOURIS(1)
}

\begin{abstract}
In this paper we show that, in order to check Gromov hyperbolicity of any surface with curvature $K \leq-k^{2}<0$, we just need to verify the Rips condition on a very small class of triangles, namely, those contained in simple closed geodesics. This result is, in fact, a new characterization of Gromov hyperbolicity for this kind of surfaces.
\end{abstract}

Key words and phrases: Gromov hyperbolicity, Riemannian surface, negatively curved Riemannian surface.

2000 AMS Subject Classification: 53C15, 53C21, 53C22, 53C23.

\section{INTRODUCTION.}

The theory of Gromov hyperbolic spaces is a useful tool in order to understand the connections between graphs and Potential Theory (see e.g. [6], [14], [19], [25], [26], [27], [28], [36], [37], [41]). Besides, the concept of Gromov hyperbolicity grasps the essence of negatively curved spaces, and has been successfully used in the theory of groups (see e.g. [20], [21], [22] and the references therein).

A geodesic metric space is called hyperbolic (in the Gromov sense) if there exists an upper bound of the distance of every point in a side of any geodesic triangle to the union of the two other sides (see Definition 2.3). This condition is known as Rips condition.

In general it is not easy to determine whether a given space is Gromov hyperbolic or not. In recent years several investigators have been interested in showing that metrics used in geometric function theory are Gromov hyperbolic. For example, the Gehring-Osgood metric ([24]) is always Gromov hyperbolic, and the Klein-Hilbert metric ([10], [29]) is also Gromov hyperbolic for certain domains. However, the Vuorinen metric ([24]) is not Gromov hyperbolic except in a particular case. Recently, Balogh and Buckley [7] have made significant progress on the hyperbolicity problem of Euclidean domains with respect to the quasihyperbolic metric (see also [12], [42] and the references therein). Another interesting instance is that of a Riemann surface endowed with the Poincaré metric. With such metric structure a Riemann surface is always negatively curved, with $K=-1$, but not every Riemann surface is Gromov hyperbolic, since topological obstacles may impede it: for instance, the two-dimensional jungle-gym (a $\mathbb{Z}^{2}$-covering of a surface of genus two) is not hyperbolic. In [4], [30], [31], [32], [38], [40] there are results about the hyperbolicity of Riemannian surfaces.

We are interested in studying when Riemannian surfaces with negative curvature are Gromov hyperbolic. The following theorem is the main result of this paper, which is a new characterization of Gromov hyperbolicity for negatively curved surfaces (see Theorem 4.1):

A Riemannian surface $S$ with $K \leq-k^{2}<0$ is hyperbolic if and only if the triangles contained in simple closed geodesics of $S$ satisfy the Rips condition.

In general, one has to check the Rips condition for all triangles. Our result states that, for negatively curved surfaces, you only have to check it for quite a smaller class of triangles.

Date: February 27, 2008.

2000 AMS Subject Classification: 53C15, 53C21, 53C22, 53C23.

(1) Research partially supported by three grants from M.E.C. (MTM 2006-13000-C03-02, MTM 2006-11976 and MTM 200626627-E), and a grant from U.C.III M./C.A.M. (CCG07-UC3M/ESP-3339), Spain. 
Furthermore, this theorem provides a bound for the hyperbolicity constant: in Riemannian surfaces with $K \leq-k^{2}<0$, if the triangles contained in simple closed geodesics satisfy the Rips condition with constant $\delta_{0}$, then every geodesic triangle also satisfies it, with constant $\delta<62 / k+\delta_{0}$.

This result is known if the curvature $K$ is constantly equal to -1 (see [39]). The argument in the proof of Theorem 4.1 is similar to the one in the proof of the constant curvature case. Unfortunately, every standard fact used in the proof in [39] is false when the curvature is not constant (in fact, the proof in [39] uses heavily hyperbolic trigonometry; however, if $K \leq-k^{2}$ we just have some trigonometric inequalities). Hence, it is necessary to prove alternative results that are valid for variable curvature. Here we develop new techniques that also improve several results in [39]. We also prove in Section 5 several consequences of Theorem 4.1, which are new in the context of variable negative curvature.

Acknowledgements. We would like to thank Professor Jose M. Rodríguez for some useful discussions. Also, we would like to thank the referee for his/her careful reading of the manuscript and for some helpful suggestions.

\section{Background in Gromov spaces and Riemannian surfaces.}

In our study of hyperbolic Gromov spaces we use the notation of [20]. We give now the basic facts about these spaces. We refer to [20] for more background and further results.

Definition 2.1. Let us fix a point $w$ in a metric space $(X, d)$. We define the Gromov product of $x, y \in X$ with respect to the point $w$ as

$$
(x \mid y)_{w}:=\frac{1}{2}(d(x, w)+d(y, w)-d(x, y)) \geq 0 .
$$

We say that the metric space $(X, d)$ is $\delta$-hyperbolic $(\delta \geq 0)$ if

$$
(x \mid z)_{w} \geq \min \left\{(x \mid y)_{w},(y \mid z)_{w}\right\}-\delta,
$$

for every $x, y, z, w \in X$. We say that $X$ is hyperbolic (in the Gromov sense) if the value of $\delta$ is not important.

It is convenient to remark that this definition of hyperbolicity is not universally accepted, since sometimes the word hyperbolic refers to negative curvature or to the existence of Green's function. However, in this paper we only use the word hyperbolic in the sense of Definition 2.1.

\section{Examples:}

(1) Every bounded metric space $X$ is (diam $X$ )-hyperbolic (see e.g. [20, p. 29]).

(2) Every complete simply connected Riemannian manifold with sectional curvature bounded from above by $-k$, with $k>0$, is hyperbolic (see e.g. [20, p. 52]).

(3) Every tree with edges of arbitrary length is 0-hyperbolic (see e.g. [20, p. 29]).

Definition 2.2. If $\gamma:[a, b] \longrightarrow X$ is a continuous curve in a metric space $(X, d)$, the length of $\gamma$ is

$$
L(\gamma):=\sup \left\{\sum_{i=1}^{n} d\left(\gamma\left(t_{i-1}\right), \gamma\left(t_{i}\right)\right): a=t_{0}<t_{1}<\cdots<t_{n}=b\right\} .
$$

We say that $\gamma$ is a geodesic if it is an isometry, i.e. $L\left(\left.\gamma\right|_{[t, s]}\right)=d(\gamma(t), \gamma(s))=|t-s|$ for every $s, t \in[a, b]$. We say that $X$ is a geodesic metric space if for every $x, y \in X$ there exists a geodesic joining $x$ and $y$; we denote by $[x, y]$ any of such geodesics (since we do not require uniqueness of geodesics, this notation is ambiguous, but convenient as well).

Definition 2.3. If $X$ is a geodesic metric space and $J$ is a polygon whose sides are $J_{1}, J_{2}, \ldots, J_{n}$, we say that $J$ is $\delta$-thin if for every $x \in J_{i}$ we have that $d\left(x, \cup_{j \neq i} J_{j}\right) \leq \delta$. If $x_{1}, x_{2}, x_{3} \in X$, a geodesic triangle $T=\left\{x_{1}, x_{2}, x_{3}\right\}$ is the union of three geodesics $\left[x_{1}, x_{2}\right],\left[x_{2}, x_{3}\right]$ and $\left[x_{3}, x_{1}\right]$. The space $X$ is $\delta$-thin (or satisfies the Rips condition with constant $\delta$ ) if every geodesic triangle in $X$ is $\delta$-thin. 
If we have a triangle with two identical vertices, we call it a "bigon". Obviously, every bigon in a $\delta$-thin space is $\delta$-thin. It is also clear that every geodesic polygon with $n$ sides in a $\delta$-thin space is $(n-2) \delta$-thin.

Definition 2.4. Given a geodesic triangle $T=\{x, y, z\}$ in a geodesic metric space $X$, let $T_{E}$ be a Euclidean triangle whose sides have the same lengths as those of $T$. Since there is no possible confusion, we will use the same notation for the corresponding points in $T$ and $T_{E}$. The maximum inscribed circle in $T_{E}$ meets the side $[x, y]$ (respectively $[y, z],[z, x]$ ) in a point $z^{\prime}$ (respectively $\left.x^{\prime}, y^{\prime}\right)$ such that $d\left(x, z^{\prime}\right)=d\left(x, y^{\prime}\right)$, $d\left(y, x^{\prime}\right)=d\left(y, z^{\prime}\right)$ and $d\left(z, x^{\prime}\right)=d\left(z, y^{\prime}\right)$. We call the points $x^{\prime}, y^{\prime}, z^{\prime}$, the internal points of $\{x, y, z\}$. There is a unique isometry $f$ of the triangle $\{x, y, z\}$ onto a tripod (a tree with one vertex $w$ of degree 3 , and three vertices $x^{\prime \prime}, y^{\prime \prime}, z^{\prime \prime}$ of degree one, such that $d\left(x^{\prime \prime}, w\right)=d\left(x, z^{\prime}\right)=d\left(x, y^{\prime}\right), d\left(y^{\prime \prime}, w\right)=d\left(y, x^{\prime}\right)=d\left(y, z^{\prime}\right)$ and $\left.d\left(z^{\prime \prime}, w\right)=d\left(z, x^{\prime}\right)=d\left(z, y^{\prime}\right)\right)$. The triangle $\{x, y, z\}$ is $\delta$-fine if $f(p)=f(q)$ implies that $d(p, q) \leq \delta$. The space $X$ is $\delta$-fine if every geodesic triangle in $X$ is $\delta$-fine.

A basic result is that hyperbolicity is equivalent to Rips condition and to the property of being fine:

Theorem 2.5. ([20, p.41]) Let us consider a geodesic metric space $X$.

(1) If $X$ is $\delta$-hyperbolic, then it is $4 \delta$-thin and $4 \delta$-fine.

(2) If $X$ is $\delta$-thin, then it is $4 \delta$-hyperbolic and $4 \delta$-fine.

(3) If $X$ is $\delta$-fine, then it is $2 \delta$-hyperbolic and $\delta$-thin.

We present now the class of maps which play the main role in the theory.

Definition 2.6. A function between two metric spaces $f: X \longrightarrow Y$ is a quasi-isometry if there are constants $a \geq 1, b \geq 0$ such that

(1) $\frac{1}{a} d_{X}\left(x_{1}, x_{2}\right)-b \leq d_{Y}\left(f\left(x_{1}\right), f\left(x_{2}\right)\right) \leq a d_{X}\left(x_{1}, x_{2}\right)+b, \quad$ for every $x_{1}, x_{2} \in X$,

(2) for any $y \in Y$, there is some $x \in X$ with $d_{Y}(y, f(x)) \leq b$.

Such a function is called an $(a, b)$-quasi-isometry. An $(a, b)$-quasigeodesic in $X$ is an $(a, b)$-quasi-isometry between an interval of $\mathbb{R}$ and $X$. An $(a, b)$-quasigeodesic segment in $X$ is an $(a, b)$-quasi-isometry between $a$ compact interval of $\mathbb{R}$ and $X$.

Notice that a quasi-isometry can be discontinuous.

Quasi-isometries are important since they are maps which preserve hyperbolicity:

Theorem 2.7. ([20, p.88]) Let us consider an $(a, b)$-quasi-isometry between two geodesic metric spaces $f: X \longrightarrow Y$. If $Y$ is $\delta$-hyperbolic, then $X$ is $\delta^{\prime}$-hyperbolic, where $\delta^{\prime}$ is a constant which only depends on $\delta$, $a$ and $b$.

Definition 2.8. Let us consider $H>0$, a metric space $X$, and subsets $Y, Z \subseteq X$. The set $V_{H}(Y):=\{x \in$ $X: d(x, Y) \leq H\}$ is called the $H$-neighborhood of $Y$ in $X$. The Hausdorff distance between $Y$ and $Z$ is defined by $\mathcal{H}(Y, Z):=\inf \left\{H>0: Y \subseteq V_{H}(Z), Z \subseteq V_{H}(Y)\right\}$.

The following is a beautiful and useful result:

Theorem 2.9. ([20, p.87]) For each $\delta \geq 0, a \geq 1$ and $b \geq 0$, there exists a constant $H=H(\delta, a, b)$ with the following property:

Let us consider a $\delta$-hyperbolic geodesic metric space $X$ and an $(a, b)$-quasigeodesic $g$ joining $x$ and $y$. If $\gamma$ is a geodesic joining $x$ and $y$, then $\mathcal{H}(g, \gamma) \leq H$.

This property is known as geodesic stability. Mario Bonk has proved that, in fact, geodesic stability is equivalent to hyperbolicity [11].

In the next sections of this paper we will work with submanifolds of Riemannian surfaces. There is a natural way to define a distance in subspaces contained in geodesic metric spaces. 
Definition 2.10. Let $X$ be a path-connected subset of a Riemannian surface. The intrinsic metric on $X$ is defined as follows:

$$
d_{X}(x, y):=\inf \{L(\gamma): \gamma \subset X \text { is a continuous curve joining } x \text { and } y\} .
$$

The following result in [38] shows that we can just deal with triangles whose sides are simple curves.

Lemma 2.11. ([38, Lemma 2.1]) Consider a geodesic metric space $X$. If every geodesic triangle in $X$ which is a simple closed curve is $\delta$-thin, then $X$ is $\delta$-thin.

Remark 2.12. This theorem allows to avoid self-intersecting triangles.

Definition 2.13. Let $(X, d)$ be a metric space, and let $\left\{X_{n}\right\}_{n} \subseteq X$ be a family of geodesic metric spaces such that $\eta_{n m}:=\eta_{m n}:=X_{n} \cap X_{m}$ are compact sets. Further, assume that for any $n$ and $m$ the set $X \backslash \eta_{n m}$ is not connected, and that $a$ and $b$ are in different connected components of $X \backslash \eta_{n m}$ for any $a \in X_{n} \backslash \eta_{n m}$, $b \in X_{m} \backslash \eta_{n m}$, with $m \neq n$. If there exist positive constants $c_{1}$ and $c_{2}$ such that $\operatorname{diam}_{X_{n}}\left(\eta_{n m}\right) \leq c_{1}$ for every $n, m$, and $d_{X_{n}}\left(\eta_{n m}, \eta_{n k}\right) \geq c_{2}$ for every $n$ and $m \neq k$, we say that $\left\{X_{n}\right\}_{n}$ is a $\left(c_{1}, c_{2}\right)$-tree decomposition of $X$.

Theorem 2.14. ([38, Theorem 2.4] and [30, Theorem 2.9]) Let us consider a metric space $X$ and a family of geodesic metric spaces $\left\{X_{n}\right\}_{n} \subseteq X$ which is a $\left(c_{1}, c_{2}\right)$-tree decomposition of $X$. Then $X$ is $\delta$-hyperbolic if and only if there exists a constant $c_{3}$ such that $X_{n}$ is $c_{3}$-hyperbolic for every $n$. Furthermore, $\delta$ (respectively $c_{3}$ ) is a universal constant which only depends on $c_{1}, c_{2}$ and $c_{3}$ (respectively $c_{1}, c_{2}$ and $\delta$ ).

Definition 2.15. Any divergent curve $\sigma:[0, \infty) \longrightarrow Y$, where $Y$ is a noncompact Hausdorff space, determines an end $E$ of $Y$. Given a compact set $F$ of $Y$, one defines $E(F)$ to be the arc component of $Y \backslash F$ that contains a terminal segment $\sigma([a, \infty))$ of $\sigma$. A set $U \subset Y$ is a neighborhood of an end $E$ if $U$ contains $E(F)$ for some compact set $F$ of $Y$. An end $E$ in a surface $S$ is collared if $E$ has a neighborhood homeomorphic to $(0, \infty) \times \mathbb{S}^{1}$. A neighborhood $U$ of $E$ will be called Riemannian collared if there exists a $C^{1}$ diffeomorphism $X:(0, \infty) \times \mathbb{S}^{1} \longrightarrow U$ such that the metric in $U$ relative to the coordinate system $X$ is written $d s^{2}=d r^{2}+G(r, \theta)^{2} d \theta^{2}$, where $G$ is a positive continuous function. We say that a closed curve $\gamma$ bounds a collared end $E$ in $S$ if some arc component of $S \backslash \gamma$ is a neighborhood of $E$.

Definition 2.16. Given a Riemannian surface $S$, a geodesic $\gamma$ in $S$, and a continuous unit vector field $\xi$ along $\gamma$, orthogonal to $\gamma$, we define the Fermi coordinates based on $\gamma$ as the map $Y(\theta, r):=\exp _{\gamma(\theta)} r \xi(\theta)$.

It is well known that the Riemannian metric can be expressed in Fermi coordinates as $d s^{2}=d r^{2}+$ $G(\theta, r)^{2} d \theta^{2}$, where $G(\theta, r)$ is the solution of the scalar equation

$$
\frac{\partial^{2} G}{\partial r^{2}}(\theta, r)+K(\theta, r) G(\theta, r)=0, \quad G(\theta, 0)=1, \quad \frac{\partial G}{\partial r}(\theta, 0)=0,
$$

(see e.g. [15, p. 247]).

Definition 2.17. A bordered surface is a surface with boundary.

$A$ halfplane is a bordered Riemannian surface which is simply connected and whose border is a single nonclosed simple geodesic.

A funnel is a bordered Riemannian surface which is a neighborhood of a collared end and whose boundary is a simple closed geodesic $\gamma$. If the curvature satisfies $K \leq-k^{2}<0$ then there is no other simple closed geodesic freely homotopic to the boundary of the funnel, and $\gamma$ minimizes the length in its free homotopy class. 


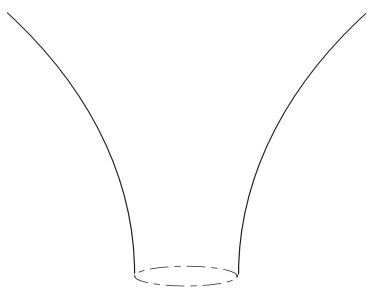

(a) Funnel

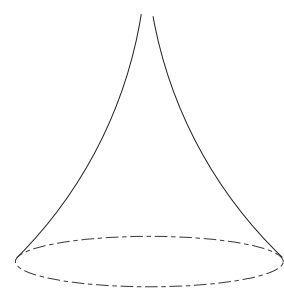

(b) Puncture

A puncture is a collared end whose fundamental group is generated by a simple closed curve $\sigma$ and there is no closed geodesic $\gamma \in[\sigma]$. If the curvature satisfies $K \leq-k^{2}<0$ then $L([\sigma])=\inf _{\gamma \in[\sigma]} L(\gamma)=0$.

$A$ bordered or nonbordered surface is doubly connected if its fundamental group is isomorphic to $\mathbb{Z}$. Every funnel and every neighborhood of a puncture are doubly connected surfaces.

A $Y$-piece is a bordered Riemannian surface which is homeomorphic to a sphere minus three open disks and whose boundary curves are simple closed geodesics. If $K=-k^{2}<0$, given three positive numbers a, $b, c$, there is a unique (up to isometry) $Y$-piece such that their boundary curves have lengths a, b, c (see e.g. [35, p.410]). Y-piece is a standard tool for constructing Riemannian surfaces with negative curvature. A clear description of these $Y$-pieces and their use are given in [13, chapter 1] and [15, chapter X.3].

A generalized $Y$-piece is a Riemannian surface (with or without boundary) which is homeomorphic to a sphere without $n$ open disks and $m$ points, with integers $n, m \geq 0$ such that $n+m=3$, the $n$ boundary curves are simple closed geodesics and the $m$ deleted points are punctures. Observe that a generalized $Y$-piece is topologically the union of a $Y$-piece and $m$ cylinders.

The following result assures that if $K \leq-k^{2}<0$, there always exists a closed geodesic in every free homotopy class, except for punctures, in which case it is impossible to have one.

Theorem 2.18. ([34, Theorem 3.7]) Let $S$ be a complete Riemannian surface with $K \leq-k^{2}<0$. Assume the boundary of $S$ either is empty or a (finite or infinite) union of pairwise disjoint simple closed geodesics. Let $\alpha$ be a homotopically nontrivial closed curve in $S$. Then there exists a minimizing closed geodesic $\gamma \in[\alpha]$ if and only if $L_{S}([\alpha])>0$.

Remark 2.19. The conclusion of this Theorem does not hold if we replace the hypothesis $K \leq-k^{2}<0$ by the weaker one $K<0$, as shown by the revolution surface of the graph of $f(x)=1+e^{x}$ around the horizontal axis (with the standard metric induced by the Euclidean metric in $\mathbb{R}^{3}$ ).

\section{TEChNicAl Lemmas.}

For the proof of Lemma 3.3 we will need to compare two functions $G_{1}$ and $G_{2}$ as in (2.1) corresponding to two different metrics. In order to achieve our objective, we prove now some technical lemmas:

Lemma 3.1. Let us consider the positive function $G(\theta, r)$ which is the solution of the equation (2.1). If $K \leq-k^{2}<0$, then $G(\theta, r) \geq \cosh k r$ for every $\theta, r \in \mathbb{R}$.

Proof. According to the equation (2.1), and bearing in mind that $G(\theta, r)>0$, we have that $\partial^{2} G / \partial r^{2}=$ $-K G \geq k^{2} G>0$ for every $\theta, r \in \mathbb{R}$. This fact implies that $\partial G / \partial r$ is an increasing function in $r \in \mathbb{R}$ for each fixed $\theta$.

If $r \geq 0$ then $\partial G / \partial r \geq 0$ and we can deduce 


$$
\begin{aligned}
\frac{\partial^{2} G}{\partial r^{2}}(\theta, r) & \geq k^{2} G(\theta, r), \\
\frac{\partial^{2} G}{\partial r^{2}}(\theta, r) \frac{\partial G}{\partial r}(\theta, r) & \geq k^{2} G(\theta, r) \frac{\partial G}{\partial r}(\theta, r), \\
\frac{\partial G}{\partial r}(\theta, r)^{2}-\frac{\partial G}{\partial r}(\theta, 0)^{2} & \geq k^{2}\left(G(\theta, r)^{2}-G(\theta, 0)^{2}\right), \\
\frac{\partial G}{\partial r}(\theta, r)^{2} & \geq k^{2}\left(G(\theta, r)^{2}-1\right), \\
\frac{\partial G / \partial r(\theta, r)}{\sqrt{G(\theta, r)^{2}-1}} & \geq k \\
\operatorname{Arcosh} G(\theta, r) & \geq k r .
\end{aligned}
$$

If $r<0$ then $\partial G / \partial r<0$, and an argument similar to the above gives the same inequality.

In both cases we have obtained the desired result: $G(\theta, r) \geq \cosh k r$ for every $\theta, r \in \mathbb{R}$.

Lemma 3.2. Let us consider $\mathbb{R}^{2}=\{(\theta, r): \theta, r \in \mathbb{R}\}$ with two different metrics given in Fermi coordinates as $d s_{1}^{2}=d r^{2}+G_{1}(\theta, r)^{2} d \theta^{2}$ and $d s_{2}^{2}=d r^{2}+G_{2}(\theta, r)^{2} d \theta^{2}$, such that their respective curvatures, $K_{1}$ and $K_{2}$, satisfy $K_{1} \leq K_{2}=-k^{2}<0$. Let us consider two curves $\sigma_{1}$ and $\sigma_{2}$ in $\mathbb{R}^{2}$ with the same endpoints, such that $\sigma_{i}$ is a geodesic for $d s_{i}(i=1,2)$. Then, $L_{d s_{1}}\left(\sigma_{1}\right) \geq L_{d s_{2}}\left(\sigma_{2}\right)$.

Proof. Let us notice that $G_{2}(\theta, r):=\cosh k r$. By Lemma 3.1, we have that $G_{1} \geq G_{2}$ for every $(\theta, r) \in \mathbb{R}^{2}$. Hence, if $\sigma_{1}(\theta)=(\theta, r(\theta))$ with $\theta \in[a, b]$,

$L_{d s_{1}}\left(\sigma_{1}\right)=\int_{\sigma_{1}} d s_{1}=\int_{a}^{b} \sqrt{r^{\prime}(\theta)^{2}+G_{1}(\theta, r(\theta))^{2}} d \theta \geq \int_{a}^{b} \sqrt{r^{\prime}(\theta)^{2}+G_{2}(\theta, r(\theta))^{2}} d \theta=L_{d s_{2}}\left(\sigma_{1}\right) \geq L_{d s_{2}}\left(\sigma_{2}\right)$.

Lemma 3.3. Let us consider a simply connected locally geodesic quadrilateral $Q$ in a complete Riemannian surface $S$ with curvature $K \leq-k^{2}<0$, whose sides $A, B, C$ and $\eta$ have respective lengths $a, b, c$ and $l$, and such that $C$ meets orthogonally the sides $A$ and $B$. Then there exists a positive constant $\lambda:=\log (5+2 \sqrt{6})$ such that $a+b+c-\lambda / k \leq l$, for every $c \geq \lambda / k$.

Remark 3.4. Let us notice that we have that $l \leq a+b+c$ by the triangle inequality.

Proof. First of all, let us recall that any trigonometric formula with $K=-1$ involving lengths $x_{1}, \ldots, x_{n}$ holds for $K=-k^{2}$ if we replace $x_{1}, \ldots, x_{n}$ by $k x_{1}, \ldots, k x_{n}$, respectively.

We start the proof assuming that $C$ an $\eta$ are disjoint; then the quadrilateral $Q$ is the boundary of a simply connected open set. Using Fermi coordinates based on $C$, we represent $C$ as the Euclidean segment joining $(0,0)$ and $(c, 0), A$ as the Euclidean segment joining $(0,0)$ and $(0, a)$, and $B$ as the Euclidean segment joining $(c, 0)$ and $(c, b)$; the side $\eta$ is the geodesic joining $(0, a)$ and $(c, b)$. Now, let us consider a simply connected locally geodesic quadrilateral $Q^{\prime}$ in a complete Riemannian surface $S$ with curvature $K=-k^{2}<0$, whose sides $A^{\prime}, B^{\prime}, C^{\prime}$ and $\eta^{\prime}$ have respective lengths $a, b, c$ and $l^{\prime}$, and such that $C^{\prime}$ meets orthogonally the sides $A^{\prime}$ and $B^{\prime}$. Applying the same before construction, it is, using Fermi coordinates, based now on $C^{\prime}$, we can represent $C^{\prime}$ as the Euclidean segment joining $(0,0)$ and $(c, 0), A^{\prime}$ as the Euclidean segment joining $(0,0)$ and $(0, a)$, and $B^{\prime}$ as the Euclidean segment joining $(c, 0)$ and $(c, b)$; the side $\eta^{\prime}$ is the geodesic joining $(0, a)$ and $(c, b)$, with length $l_{0}$. By Lemma 3.2 , we know that $L_{S}(\eta)=l \geq l_{0}$.

We are going to use the next hyperbolic trigonometry formula with constant curvature $K=-k^{2}<0$ (see $\left[18\right.$, p. 88]) and the comparation of lengths $l \geq l_{0}$ :

$$
\cosh k l \geq \cosh k l_{0}=\cosh k a \cosh k b \cosh k c-\sinh k a \sinh k b .
$$


Using now the facts that, on the one hand, $2(\cosh t-1) e^{-t}=\left(1-e^{-t}\right)^{2}$ and, on the other hand, for $\lambda:=\log (5+2 \sqrt{6})$ it is satisfied the equality $\frac{1}{8}\left(1-e^{-\lambda}\right)^{2}=e^{-\lambda}$, we have:

$$
\begin{aligned}
e^{k l} & \geq \cosh k l \geq \cosh k a \cosh k b \cosh k c-\sinh k a \sinh k b \geq(\cosh k c-1) \cosh k a \cosh k b \\
& =\frac{1}{2}\left(1-e^{-k c}\right)^{2} e^{k c} \cosh k a \cosh k b \geq \frac{1}{8}\left(1-e^{-k c}\right)^{2} e^{k(a+b+c)} \geq \frac{1}{8}\left(1-e^{-\lambda}\right)^{2} e^{k(a+b+c)} \\
& =e^{-\lambda} e^{k(a+b+c)},
\end{aligned}
$$

for every $c \geq \lambda / k$. Therefore $l \geq a+b+c-\lambda / k$.

If $C$ an $\eta$ are not disjoint we use the hyperbolic trigonometry formula with constant curvature $K=-k^{2}<$ 0 (see $[18$, p. 88]) and, once again, Fermi coordinates and Lemma 3.2 give:

$\cosh k l \geq \cosh k l_{0}=\cosh k a \cosh k b \cosh k c+\sinh k a \sinh k b$.

By a similar argument to the previous one:

$$
e^{k l} \geq \cosh k l \geq \cosh k a \cosh k b \cosh k c+\sinh k a \sinh k b \geq \cosh k c \cosh k a \cosh k b \geq \frac{1}{8} e^{k(a+b+c)},
$$

with no restriction about the length $c$.

Notice that in this case we obtain a sharper inequality $a+b+c-(3 \log 2) / k \leq l$.

Lemma 3.5. Let us consider a simply connected locally geodesic quadrilateral $Q$ in a complete Riemannian surface $S$ with curvature $K \leq-k^{2}<0$, with sides $A, B, C$ and $\eta$, such that $C$ meets orthogonally the sides $A$ and $B$, and $L_{S}(C) \geq \lambda / k$ with $\lambda:=\log (5+2 \sqrt{6})$. We also assume that $\eta$ is a geodesic in $S$. If we define $\sigma:=A \cup C \cup B$, then the curve $\sigma$ is a $(1, \lambda / k)$-quasigeodesic with its arc-length parametrization.

Proof. Denoting by $a, b, c$ and $l$ the lengths of the sides $A, B, C$ and $\eta$ respectively, since $\sigma:[0, a+b+c] \rightarrow S$ is a continuous curve, it is clear that $d(\sigma(t), \sigma(s)) \leq L_{S}(\sigma([t, s]))=|t-s|$ always. Without loss of generality, from now on we are assuming that $t<s$.

We are going to prove that $|t-s| \leq d(\sigma(t), \sigma(s))+\lambda / k$. In order to prove it, let us assume that it is false, and let us seek a contradiction. Suppose then that $|t-s|>d(\sigma(t), \sigma(s))+\lambda / k$ and define a curve $\sigma_{0}$ as the union of the next three curves: $\sigma([0, t])$, a geodesic connecting $\sigma(t)$ with $\sigma(s)$, and $\sigma([s, a+b+c])$. Let us denote by $u, v$ the endpoints of $\sigma$ (notice that they are also the endpoints of the geodesic $\eta$ ); since $\sigma_{0}$ is a continuous curve connecting $u$ and $v$, we have that, applying Lemma 3.3,

$$
\begin{aligned}
d(u, v) & =L_{S}(\eta)=l \leq L_{S}\left(\sigma_{0}\right)=L_{S}(\sigma)-L_{S}(\sigma([t, s]))+d(\sigma(t), \sigma(s)) \\
& =L_{S}(\sigma)-|t-s|+d(\sigma(t), \sigma(s)) \\
& <L_{S}(\sigma)-\lambda / k=a+b+c-\lambda / k \leq l=d(u, v),
\end{aligned}
$$

which is a contradiction. Therefore we can assert that

$$
|t-s|-\lambda / k \leq d(\sigma(t), \sigma(s)) \leq|t-s|, \text { for every } 0 \leq t, s \leq a+b+c .
$$

Lemma 3.6. Given a complete Riemannian surface $S$, let us consider a continuous curve $g$ joining $u, v \in S$. If $L_{S}(g) \leq \alpha$, then $g$ is a $(1, \alpha)$-quasigeodesic with its arc-length parametrization.

Proof. Let $g:[0, l] \longrightarrow S$ with its arc-length parametrization. Since $g$ is continuous, it is clear that $d(g(t), g(s)) \leq L_{S}(g([t, s]))=|t-s|$. Let us notice now $|t-s| \leq L_{S}(g) \leq \alpha \leq d(g(t), g(s))+\alpha$.

Therefore, we can assert that

$$
|t-s|-\alpha \leq d(g(t), g(s)) \leq|t-s|, \text { for every } 0 \leq t, s \leq l .
$$


Lemma 3.7. Let $S$ be a complete Riemannian surface with $K \leq-k^{2}<0, \delta_{1}:=\frac{1}{k} \log (1+\sqrt{2})$ and let $Q$ be a simply connected locally geodesic quadrilateral in $S$. Assume the sides, $A, B, C$ and $\eta$ of $Q$ satisfy the following two conditions: $(i) L_{S}(C)>4 \delta_{1}$, (ii) $C$ meets orthogonally the sides $A$ and $B$.

Then, we have that $d(z, \eta) \leq 4 \delta_{1}$ for every $z \in C$.

Proof. To start, note that the sides $C$ and $\eta$ might meet or might not (but if they do, it must be in a single point). However we use an argument which covers both cases.

Without loss of generality we can assume that $S$ is simply connected, since otherwise we can lift $Q$ to the universal covering of $S$ (recall that $Q$ is simply connected and that the distances in the universal cover are greater than in the surface). Consequently, $Q$ is a geodesic quadrilateral (in a simply connected Riemannian surface with $K \leq-k^{2}$, every local geodesic is, in fact, a geodesic). $52])$.

Since $S$ is a simply connected and complete Rimannian surface, it is $\delta_{1}$-thin (see [2, p. 130] and [20, p.

Let us consider the geodesic $C$ as an oriented curve joining $A$ and $B$. By hypothesis $L_{S}(C)>4 \delta_{1}$; therefore we can assert that there exist two points $\alpha$ and $\beta$ (with $\alpha<\beta$ ) in the oriented geodesic $C$ defined as $\alpha:=\max \left\{z \in C: d(z, A) \leq 2 \delta_{1}\right\}$, and $\beta:=\min \left\{z \in C: d(z, B) \leq 2 \delta_{1}\right\}$.

Since $Q$ is $2 \delta_{1}$-thin, $d(z, A \cup B \cup \eta) \leq 2 \delta_{1}$ for every $z \in C$. If $z \in(\alpha, \beta) \subset C$ then $d(z, A \cup B)>2 \delta_{1}$ and, therefore, $d(z, A \cup B \cup \eta)=d(z, \eta) \leq 2 \delta_{1}$; consequently, $d(z, \eta) \leq 2 \delta_{1}$ for every $z \in[\alpha, \beta]$. If $z \in C \backslash[\alpha, \beta]$ it is verified that $d(z,[\alpha, \beta]) \leq 2 \delta_{1}$ and, therefore, $d(z, \eta) \leq 4 \delta_{1}$.

Definition 3.8. Let $\lambda$ be the constant in Lemma 3.3 and $S$ a complete Riemannian surface with $K \leq-k^{2}<$ 0 . A $\lambda$-triangle in $S$ is defined to be a triangle with continuous injective $(1, \lambda / k)$-quasigeodesic sides with its arc-length parametrization.

Lemma 3.9. Let us consider a complete Riemannian surface $S$ with $K \leq-k^{2}<0$, a simple closed geodesic $\gamma$ with length $l$ and a $\lambda$-triangle $T$ contained in $\gamma$ and homotopic to $\gamma$. Then, every side of $T$ has length less than or equal to $\frac{l}{2}+\frac{\lambda}{k}$. Furthermore, at least two of the sides of $T$ have length greater than or equal to $\frac{l}{4}-\frac{\lambda}{2 k}$.

Proof. If $T:=\{a, b, c\}$, let us denote by $l_{1}:=L_{S}([a, b]), l_{2}:=L_{S}([b, c])$ and $l_{3}:=L_{S}([a, c])$.

Seeking for a contradiction, let us assume that one side of $T$, for example $[a, c]$, is "long", i.e. $l_{3}>l / 2+\lambda / k$. However $[a, c]$ is, as a side of $T$, a $(1, \lambda / k)$-quasigeodesic with its arc-length parametrization $\sigma_{3}:\left[0, l_{3}\right] \longrightarrow \gamma$. Then, $l_{3}-\lambda / k \leq d(a, c) \leq l_{3}+\lambda / k$.

But $d(a, c) \leq l / 2$ since $a$ and $c$ belong to a closed curve with length $l$. Therefore, $l_{3}-\lambda / k \leq d(a, c) \leq l / 2$, which implies that $l_{3} \leq l / 2+\lambda / k$ and this fact contradicts the assumption about $l_{3}$ being "long".

Finally, we prove the last part of the Lemma. Seeking again for a contradiction, let us assume that two sides, for example $[a, b]$ and $[b, c]$, are "short", that is, $l_{1}, l_{2}<l / 4-\lambda /(2 k)$. Then $l_{3}>l / 2+\lambda / k$, since $l=l_{1}+l_{2}+l_{3}$, which is, in fact, a contradiction.

Lemma 3.10. Let us consider a doubly connected complete Riemannian surface $S$ with curvature $K \leq$ $-k^{2}<0$, and let $l$ be the length of the simple closed geodesic $\gamma$ in it. Then $S$ is $\left(18 \delta_{1}+2 l\right)$-thin, with $\delta_{1}:=\log (1+\sqrt{2}) / k$.

Remark 3.11. According to the above Lemma, every geodesically convex subset of a surface like the one described in it, is $\left(18 \delta_{1}+2 l\right)$-thin as well. Further, every funnel $F$ in a Riemanian surface with $K \leq-k^{2}<0$ and $L_{S}(\partial F)=l$ is $\left(18 \delta_{1}+2 l\right)$-thin. 
Proof. Let us consider a geodesic triangle $T=\{a, b, c\}$ in $S$. By Lemma 2.11 we can assume that $T$ is a simple closed curve. Notice that $T$ is homotopic to either to a point or the simple closed geodesic $\gamma$. If $T$ is homotopic to a point, then it is the boundary of a simply connected closed set $E$, and consequently $E$, with its intrinsic distance, is isometric to some subset of the universal covering surface $\widetilde{S}$; this implies that $T$ is $\delta_{1}$-thin (recall that $\widetilde{S}$ is $\delta_{1}$-thin). Therefore, to finish the proof we may assume that $T$ is homotopic to the simple closed geodesic $\gamma$ of $S$.

In this case, there are two possible situations:

(1) Two of the vertices of the triangle coincide. Then $T$ is a geodesic bigon whose two sides are two geodesics $\gamma_{1}$ and $\gamma_{2}$ with the same length. Now, we have Figure 1 (drawn in the universal covering surface). In it, the vertices $a$ and $a_{0}$ must be considered as coincident, but we have maintained both names to be able to identify clearly $\gamma_{1}$ with $[a, b]$ and $\gamma_{2}$ with $\left[b, a_{0}\right]$. Let us call $a^{\prime}, b^{\prime}$ and $a_{0}^{\prime}$ their respective projections over $\gamma$ (where $\left[a^{\prime}, b^{\prime}\right]$ is the projection of $\gamma_{1}$ over $\gamma$ and $\left[b^{\prime}, a_{0}^{\prime}\right]$ is the projection of $\gamma_{2}$ over $\gamma$; although all the projections are obviously orthogonal, in the Figure 1 we have not used right angles to emphasize that the lengths of the segments $\left[a^{\prime}, b^{\prime}\right]$ and $\left[b^{\prime}, a_{0}^{\prime}\right]$ might be very different, since the curvature is not constant).

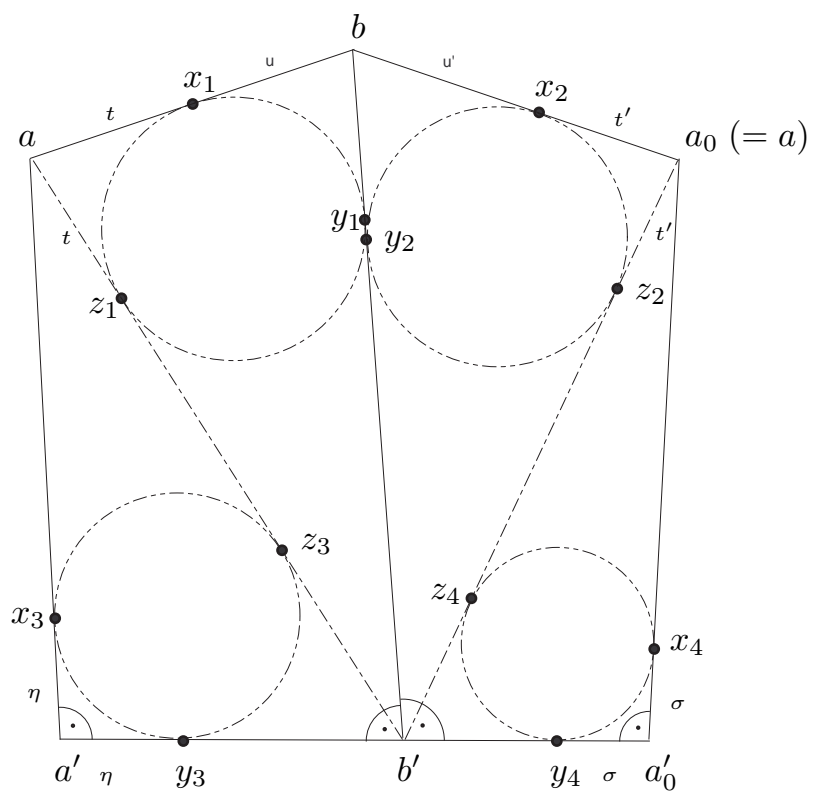

Figure 1. $T$ is a geodesic bigon.

Let us draw now the geodesics $\left[a, b^{\prime}\right]$ and $\left[a_{0}, b^{\prime}\right]$ and let $l_{1}$ and $l_{2}$ be their respective lengths. Next, we are going to prove that $\left|l_{1}-l_{2}\right| \leq 3 l$. In order to do it, we need to consider the following four geodesic triangles: $T_{1}=\left\{a, b, b^{\prime}\right\}$ (with internal points $x_{1}, y_{1}, z_{1}$ ), $T_{2}=\left\{b, a_{0}, b^{\prime}\right\}$ (with internal points $\left.x_{2}, y_{2}, z_{2}\right), T_{3}=\left\{a, a^{\prime}, b^{\prime}\right\}$ (with internal points $x_{3}, y_{3}, z_{3}$ ) and $T_{4}=\left\{a_{0}, a_{0}^{\prime}, b^{\prime}\right\}$ (with internal points $\left.x_{4}, y_{4}, z_{4}\right)$. Notice that $T_{1}, T_{2}, T_{3}$ and $T_{4}$ are, all of them, $4 \delta_{1}$-fine, because they are homotopically trivial and then their lifts to the universal covering surface are $4 \delta_{1}$-fine.

If we denote $\eta:=L_{S}\left(\left[a^{\prime}, x_{3}\right]\right)=L_{S}\left(\left[a^{\prime}, y_{3}\right]\right)$ and $\sigma:=L_{S}\left(\left[a_{0}^{\prime}, x_{4}\right]\right)=L_{S}\left(\left[a_{0}^{\prime}, y_{4}\right]\right)$ then

$l_{1}=L_{S}\left(\left[a, z_{3}\right]\right)+L_{S}\left(\left[z_{3}, b^{\prime}\right]\right)=L_{S}\left(\left[a, x_{3}\right]\right)+L_{S}\left(\left[b^{\prime}, y_{3}\right]\right)=L_{S}\left(\left[a, a^{\prime}\right]\right)+L_{S}\left(\left[a^{\prime}, b^{\prime}\right]\right)-2 \eta$, and $l_{2}=L_{S}\left(\left[a_{0}, z_{4}\right]\right)+L_{S}\left(\left[z_{4}, b^{\prime}\right]\right)=L_{S}\left(\left[a_{0}, x_{4}\right]\right)+L_{S}\left(\left[b^{\prime}, y_{4}\right]\right)=L_{S}\left(\left[a_{0}, a_{0}^{\prime}\right]\right)+L_{S}\left(\left[a_{0}^{\prime}, b^{\prime}\right]\right)-2 \sigma$. 
Hence:

$$
\begin{aligned}
\left|l_{2}-l_{1}\right| & =\left|L_{S}\left(\left[a_{0}, a_{0}^{\prime}\right]\right)+L_{S}\left(\left[a_{0}^{\prime}, b^{\prime}\right]\right)-2 \sigma-L_{S}\left(\left[a, a^{\prime}\right]\right)-L_{S}\left(\left[a^{\prime}, b^{\prime}\right]\right)+2 \eta\right| \\
& =\left|L_{S}\left(\left[a_{0}^{\prime}, b^{\prime}\right]\right)-L_{S}\left(\left[a^{\prime}, b^{\prime}\right]\right)-2(\sigma-\eta)\right| \leq 3 l .
\end{aligned}
$$

Now we claim that both $\left|d\left(a_{0}, x_{2}\right)-d\left(a, x_{1}\right)\right| \leq 2 l$ and $\left|d\left(b, x_{1}\right)-d\left(b, x_{2}\right)\right| \leq 2 l$. For simplicity of notation we are going to give names to the lengths involved in the proof. So, we define $l_{3}:=L_{S}([a, b])=L_{S}\left(\left[b, a_{0}\right]\right), l_{4}:=L_{S}\left(\left[b, b^{\prime}\right]\right), u:=L_{S}\left(\left[b, x_{1}\right]\right), u^{\prime}:=L_{S}\left(\left[b, x_{2}\right]\right), t:=L_{S}\left(\left[a, x_{1}\right]\right)=$ $L_{S}\left(\left[a, z_{1}\right]\right), t^{\prime}:=L_{S}\left(\left[a_{0}, x_{2}\right]\right)=L_{S}\left(\left[a_{0}, z_{2}\right]\right), s:=L_{S}\left(\left[b^{\prime}, z_{1}\right]\right)$ and $s^{\prime}:=L_{S}\left(\left[b^{\prime}, z_{2}\right]\right)$.

Since $l_{1}+l_{2}+l_{3}=2 s+2 t+2 u$ and $l_{1}=s+t$, subtracting both equations we get $u=l_{3} / 2+l_{4} / 2-l_{1} / 2$. Applying a similar argument, we can also obtain $u^{\prime}=l_{3} / 2+l_{4} / 2-l_{2} / 2$. Hence,

$$
\begin{aligned}
\left|d\left(b, x_{1}\right)-d\left(b, x_{2}\right)\right| & =\left|u-u^{\prime}\right|=\left|l_{2}-l_{1}\right| / 2 \leq 3 l / 2<2 l, \text { and } \\
\left|d\left(a_{0}, x_{2}\right)-d\left(a, x_{1}\right)\right| & =\left|t^{\prime}-t\right|=\left|l_{3}-u^{\prime}-l_{3}+u\right|=\left|u-u^{\prime}\right| \leq 3 l / 2<2 l
\end{aligned}
$$

as we claimed.

Now we proceed to show that our bigon $T$ is $\left(16 \delta_{1}+2 l\right)$-thin. To do it, let us take an arbitrary point $z \in T$. Obviously there is no loss of generality in assuming that $z \in \gamma_{1}$. More precisely, $z$ may belong either to the segment $\left[b, x_{1}\right]$ or to the segment $\left[a, x_{1}\right]$. In the first case, there are two possible situations:

(a) $z$ is a point such that $d(b, z) \leq \min \left\{u, u^{\prime}\right\}$. Then, since $T_{1}$ is $4 \delta_{1}$-fine, there exists a point $z^{\prime} \in\left[b, y_{1}\right]$ such that $d\left(z, z^{\prime}\right) \leq 4 \delta_{1}$. Applying the same argument to the $4 \delta_{1}$-fine triangle $T_{2}$, there exists a point $z^{\prime \prime} \in\left[b, x_{2}\right]$ such that $d\left(z^{\prime}, z^{\prime \prime}\right) \leq 4 \delta_{1}$. It means that we have been able to find a point $z^{\prime \prime} \in \gamma_{2}$ verifying that $d\left(z, z^{\prime \prime}\right) \leq d\left(z, z^{\prime}\right)+d\left(z^{\prime}, z^{\prime \prime}\right) \leq 8 \delta_{1}$.

(b) $z$ is a point such that $d(b, z)>\min \left\{u, u^{\prime}\right\}$. If this is so, the first thing to do is getting another point $z_{0} \in\left[b, x_{1}\right]$ such that $d\left(b, z_{0}\right) \leq \min \left\{u, u^{\prime}\right\}$ and then apply the previous case to that $z_{0}$. Notice that, as $\left|u-u^{\prime}\right| \leq 2 l$ by $(3.2), z_{0}$ can be found easily by moving to the right at most a distance $2 l$ along $\gamma_{1}$ starting at $x_{1}$. Then, by the arguments used before, we are able to find a point $z^{\prime \prime} \in \gamma_{2}$ such that $d\left(z, z^{\prime \prime}\right) \leq d\left(z, z_{0}\right)+d\left(z_{0}, z^{\prime}\right)+d\left(z^{\prime}, z^{\prime \prime}\right) \leq 2 l+8 \delta_{1}$.

Now, if $z \in\left[a, x_{1}\right]$ we repeat the same arguments but taking into account that besides $T_{1}$ and $T_{2}$, the triangles $T_{3}$ and $T_{4}$ are $4 \delta_{1}$-fine as well (recall that by $(3.2)$ we also have $\left|t^{\prime}-t\right| \leq 2 l$ ). Hence, we know that there exists a point $z^{\prime \prime} \in \gamma_{2}$ such that $d\left(z, z^{\prime \prime}\right) \leq 16 \delta_{1}+2 l$, which implies our conclusion.

(2) Now, let $T$ be a general geodesic triangle, with its three vertices different. See Figure 2, in which we have unfolded our triangle in the universal covering surface to make things more understandable. There we have used two different names $a$ and $a_{0}$ for the same vertex in order to simplify notation in the following argument (they are identified when the triangle is "glued" again).

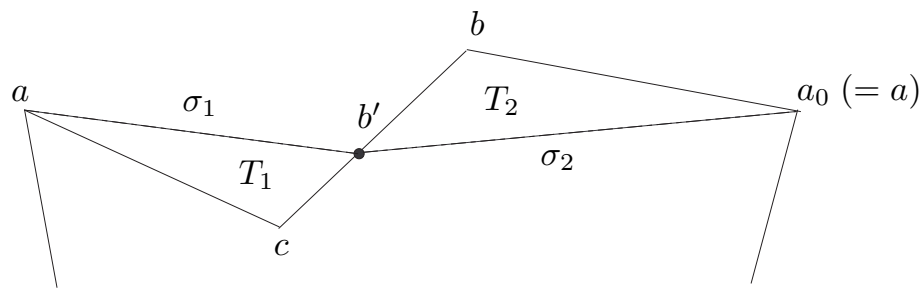

Figure 2. $T$ is a general triangle. 
Let us define $\Gamma$ as the set of curves joining $c$ and $a$ which are homotopic to $[c, b] \cup[b, a]$, and $\Gamma^{\prime}$ as the set of curves joining $a$ and $b$ which are homotopic to $[a, c] \cup[c, b]$. It is obvious that the distance from $c$ to $a$ (that is to say, the length of the geodesic side $[a, c]$ ) is less than or equal to the distance between the same two points when we just consider the curves in $\Gamma$. That is to say, $d(c, a) \leq d_{\Gamma}(c, a)$. For the sake of simplicity in the notation of the following argument, we will denote $d_{\Gamma}(c, a)$ by $d\left(c, a_{0}\right)$ (see Figure 2 for a better understanding).

A similar argument yields $d_{\Gamma^{\prime}}(a, b) \geq d(b, a)$. Again, in order to simplify the notation (and to maintain the coherence with Figure 2), we will denote $d_{\Gamma^{\prime}}(a, b)$ simply by $d(a, b)$, and we will refer to the length of the geodesic side $[b, a]$ as $d\left(b, a_{0}\right)$.

Let us define now a function $f(x):=d(x, a)-d\left(x, a_{0}\right)$. Since $f(c)=d(c, a)-d\left(c, a_{0}\right) \leq 0$ and $f(b)=d(b, a)-d\left(b, a_{0}\right) \geq 0$, it means that there must exist a point $b^{\prime} \in[b, c]$ such that $f\left(b^{\prime}\right)=d\left(b^{\prime}, a\right)-d\left(b^{\prime}, a_{0}\right)=0$.

Notice that there are two nonhomotopic geodesics joining the vertex $a$ with the point $b^{\prime}$. Let $\sigma_{1}$ be the geodesic homotopic to $[a, c] \cup\left[c, b^{\prime}\right] ; \sigma_{2}$ the geodesic homotopic to $\left[b^{\prime}, b\right] \cup\left[b, a_{0}\right]$. Then $\sigma_{1} \cup \sigma_{2}$ is a geodesic bigon whose sides have the same length. If we apply the previous case to this bigon, we can state that it is $\left(16 \delta_{1}+2 l\right)$-thin.

Next, let us consider the two simply connected triangles $T_{1}=[a, c] \cup\left[b^{\prime}, c\right] \cup \sigma_{1}$ and $T_{2}=\left[a_{0}, b\right] \cup$ $\left[b, b^{\prime}\right] \cup \sigma_{2}$, both of them $\delta_{1}$-thin. So, given any point $z \in[a, c]$, there exists a point $z^{\prime} \in\left[b^{\prime}, c\right] \cup \sigma_{1}$ with $d\left(z, z^{\prime}\right) \leq \delta_{1}$. If $z^{\prime} \in\left[b^{\prime}, c\right] \subset[b, c]$ we have finished the proof, and we conclude that $d(z, A) \leq \delta_{1}$. If $z^{\prime} \in \sigma_{1}$, we know that there exists a point $z^{\prime \prime} \in \sigma_{2}$ such that $d\left(z^{\prime}, z^{\prime \prime}\right) \leq 16 \delta_{1}+2 l$ since the bigon $\sigma_{1} \cup \sigma_{2}$ is $\left(16 \delta_{1}+2 l\right)$-thin. Hence, $d\left(z, \sigma_{2}\right) \leq 17 \delta_{1}+2 l$; since $T_{2}$ is also $\delta_{1}$-thin, it means that $d(z, A) \leq 18 \delta_{1}+2 l$, and therefore our general triangle $T$ is $\left(18 \delta_{1}+2 l\right)$-thin, which finishes the proof.

Corollary 3.12. Let us consider a doubly connected complete Riemannian surface $S$ with no simple closed geodesic, and with curvature $K \leq-k^{2}<0$. Then $S$ is $18 \delta_{1}$-thin, with $\delta_{1}:=\log (1+\sqrt{2}) / k$.

Remark 3.13. According to the above Corollary, every geodesically convex subset of a surface like the one described in it, is $18 \delta_{1}$-thin as well.

Proof. As the proof is almost identical to that of Lemma 3.10, we will just explain the only difference. Let us consider a geodesic bigon $T=\{a, b\}$ as in the proof of Lemma 3.10. Since there is no simple closed geodesic in $S$ homotopic to $T$, and the curvature $K$ is strictly negative, there exist non-trivial closed curves with arbitrarily small length which are homotopic to $T$. For any fixed $\varepsilon>0$ let us choose one of these closed curves with length smaller than $\varepsilon$ and let us call it $\sigma_{0}$. Then, we can repeat the same construction used in the proof of Lemma 3.10 for geodesic bigons, but projecting the vertices $a$ and $b$ of $T$ over $\sigma_{0}$ rather than over a simple closed geodesic (which does not exist in our situation). Let us call $a^{\prime}$ and $b^{\prime}$ the respective projections of $a$ and $b$ over $\sigma_{0}$. It is clear that there exist two non-homotopic geodesic segments $\sigma_{1}$ and $\sigma_{2}$ joining $a^{\prime}$ and $b^{\prime}$. We choose now $\sigma=\sigma_{1} \cup \sigma_{2}$. Then we can repeat the same arguments used in the proof of Lemma 3.10 replacing $\left[a^{\prime}, b^{\prime}\right]$ and $\left[b^{\prime}, a_{0}^{\prime}\right]$ by $\sigma_{1}$ and $\sigma_{2}$, and conclude that the bigon $T$ is $\left(16 \delta_{1}+2 \varepsilon\right)$-thin.

Applying this result to a general geodesic triangle $T$ as in the proof of Lemma 3.10, we can conclude that $T$ is $\left(18 \delta_{1}+2 \varepsilon\right)$-thin.

Since $\varepsilon$ can be arbitrarily small, a passage to the limit (making $\varepsilon \rightarrow 0$ ) implies that our general triangle is also $18 \delta_{1}$-thin. 


\section{The MAIN RESUlt.}

Theorem 4.1. Let $S$ be a complete Riemannian surface with $K \leq-k^{2}<0$ (with or without boundary); if $S$ has boundary, we also require that $\partial S$ is the union of local geodesics (closed or non-closed). Let $\lambda:=$ $\log (5+2 \sqrt{6})$; then $S$ is $\delta$-hyperbolic if and only if every $\lambda$-triangle contained in a simple closed geodesic of $S$ is $\delta_{0}$-thin.

More precisaly, if $S$ is $\delta$-thin, then every $\lambda$-triangle contained in a simple closed geodesic in $S$ is $\delta_{0}$-thin with $\delta_{0}=\delta+2 H$, where $H=H(4 \delta, 1, \lambda / k)$ is the constant in Theorem 2.9; and if every $\lambda$-triangle contained in a simple closed geodesic in $S$ is $\delta_{0}$-thin then $S$ is $\delta$-thin, with $\delta:=\max \left\{18 \delta_{1}+20 \lambda / k, 12 \delta_{1}+\delta_{0}\right\}$ and $\delta_{1}:=\frac{1}{k} \log (1+\sqrt{2})$.

Remark 4.2. Let us notice that, as $\log (1+\sqrt{2}) \approx 0.8814$ and $\lambda:=\log (5+2 \sqrt{6}) \approx 2.2924$, the hyperbolicity constant $\delta$ can be bounded in the following way: $\delta \leq \delta_{0}+18 \delta_{1}+20 \lambda / k<\delta_{0}+62 / k$

Proof. Let us assume first that $S$ is $\delta$-hyperbolic and let us check that every $\lambda$-triangle contained in a simple closed geodesic of $S$ is $\delta_{0}$-thin, with $\delta_{0}=\delta+2 H$. Let $T$ be a $\lambda$-triangle with sides $g_{1}, g_{2}, g_{3}$, contained in a simple closed geodesic $\gamma$. Theorem 2.9 implies that for every quasigeodesic side $g_{i}$ there exists a geodesic $\gamma_{i}$ with the same endpoints as $g_{i}$, such that $\mathcal{H}\left(g_{i}, \gamma_{i}\right) \leq H=H(4 \delta, 1, \lambda / k)$. We have to prove that the distance from any point in a side of $T$ to the union of the other two sides is bounded from above by $\delta_{0}$. Without loss of generality let us assume that $z \in g_{1}$. Hence, for every $z \in g_{1}$, there is a point $z_{0} \in \gamma_{1}$ with $d\left(z, z_{0}\right) \leq H$. Since $S$ is $\delta$-thin, we can find $z_{0}^{\prime} \in \gamma_{2} \cup \gamma_{3}$ with $d\left(z_{0}, z_{0}^{\prime}\right) \leq \delta$. Finally, we also have a point $z^{\prime} \in g_{2} \cup g_{3}$ with $d\left(z^{\prime}, z_{0}^{\prime}\right) \leq H$. Consequently $d\left(z, g_{2} \cup g_{3}\right) \leq d\left(z, z^{\prime}\right) \leq \delta+2 H$.

Now, let us assume that every $\lambda$-triangle contained in a simple closed geodesic of $S$ is $\delta_{0}$-thin and let us prove that $S$ is $\delta$-hyperbolic.

First, notice that if $S$ has boundary, the hypothesis implies that $\partial S$ is the union of pairwise disjoint simple local geodesics (closed or non-closed). In this case, we can construct a complete Riemannian surface $R$ without boundary and with $K \leq-k^{2}$ by pasting to $S$ a cylinder along each simple closed geodesic, and a halfplane in each non-closed simple geodesic. If $\gamma_{0} \subseteq \partial S$ is a closed geodesic with length $l$, we can consider the Fermi coordinates based on $\gamma_{0}$. The Riemannian metric can be expressed in Fermi coordinates as $d s^{2}=d r^{2}+G(\theta, r)^{2} d \theta^{2}$, with $G(\theta, r)$ satisfying $(2.1)$, where the function $K(\theta, r)$ is $C^{\infty}$ in $\mathbb{R} \times(-\infty, 0]$ and $l$-periodic in $\theta$; actually, $K(\theta, r) \in C^{\infty}(\mathbb{R} \times(-\infty, 3 \varepsilon))$, for some $\varepsilon>0$. Let $\psi(r) \in C^{\infty}(\mathbb{R})$ be a function satisfying

$$
\psi(r)= \begin{cases}1, & r \leq \varepsilon, \\ 0, & r \geq 2 \varepsilon,\end{cases}
$$

and $0 \leq \psi(r) \leq 1$. We define $\widetilde{K}(\theta, r):=K(\theta, r) \psi(r)-(1-\psi(r)) k^{2} ;$ then $\widetilde{K}(\theta, r)=K(\theta, r)$ in $\mathbb{R} \times(-\infty, \varepsilon]$ and $\widetilde{K}(\theta, r)=-k^{2}$ in $\mathbb{R} \times[2 \varepsilon, \infty)$. Therefore, $\widetilde{K}(\theta, r)$ is $C^{\infty}$ in $\mathbb{R} \times \mathbb{R}, l$-periodic in $\theta$ and $\widetilde{K} \leq-k^{2}$. Now we consider the function $\widetilde{G}(\theta, r)$ associated to $\widetilde{K}(\theta, r)$ and satisfying (2.1). This allows to attach a cylinder to $S$ along each simple closed geodesic $\gamma_{0} \subseteq \partial S$. If $\gamma_{0} \subseteq \partial S$ is a non-closed geodesic we can apply a similar argument to the previous one. We must consider another function $\psi(\theta, r)$ depending on $\theta$ and $r$ since, in this case, $G$ need not be periodic. This allows to attach a halfplane to $S$ along each simple non-closed geodesic $\gamma_{0} \subseteq \partial S$

In any case we get a complete Riemannian surface $R$ containing $S$ and with curvature less than or equal to $-k^{2}$. Since $S$ is geodesically convex in $R$ (every geodesic connecting two points of $S$ is contained in $S$ ), then $d_{R}(z, w)=d_{S}(z, w)$ for every $z, w \in S$, and any simple closed geodesic in $R$ is contained in $S$. Therefore, it is sufficient to prove the Theorem for surfaces without boundary.

Since the universal covering map, $\pi: \tilde{S} \rightarrow S$ is a local isometry, the universal covering $\tilde{S}$ (which is a simply connected and complete surface satisfying that $K \leq-k^{2}$ ) is $\delta_{1}$-thin (see [2, p.130] and [20, p.52]).

Now, let us consider a geodesic triangle $T=\{a, b, c\}$ in $S$. By Lemma 2.11, we can assume that $T$ is a simple closed curve. There are three possibilities: $T$ is homotopic to a point, $T$ is homotopic to a puncture, or $T$ is freely homotopic to a simple closed geodesic in $S$. 
If $T$ is homotopic to a point, then it is the boundary of a simply connected closed set $E$, and consequently $E$, with its intrinsic distance, is isometric to some geodesically convex subset of $\tilde{S}$; this implies that $T$ is $\delta_{1}$-thin (recall that $\tilde{S}$ is $\delta_{1}$-thin).

If $T$ is homotopic to a puncture, then it is the boundary of a closed doubly connected set, which is, with its intrinsic distance, isometric to some geodesically convex subset of a doubly connected surface verifying the hypothesis in Corollary 3.12. This implies that $T$ is $18 \delta_{1}$-thin.

Otherwise, $T=\{a, b, c\}$ is freely homotopic to a simple closed geodesic $\gamma$ in $S$.

We are going to deal with two cases.

If $L_{S}(\gamma) \leq 10 \lambda / k$, we consider a doubly connected complete Riemannian surface, $S_{0}$, with $K \leq-k^{2}<0$ containing a simple closed geodesic $\tilde{\gamma}$ of length $l$, such that the closed set in $S$ bounded by $T$ and $\gamma$ is, with its intrinsic distance, isometric to a subset in $S_{0}$, bounded by $\tilde{\gamma}$ and a triangle $T_{0}$. We know that $S_{0}$ is $18 \delta_{1}+2 l$-thin by Lemma 3.10 . These facts imply that $T$ is $\left(18 \delta_{1}+20 \lambda / k\right)$-thin.

We consider now the case $L_{S}(\gamma)>10 \lambda / k$.

The main idea of the proof is to project the three vertices of $T$ onto the simple closed geodesic $\gamma$ to obtain a new triangle $T^{\prime} \subseteq \gamma$. We will check that $T^{\prime}$ is a $\lambda$-triangle and hence it is $\delta_{0}$-thin by hypothesis.

Let $a^{\prime}, b^{\prime}$ and $c^{\prime}$ be the projections over the geodesic $\gamma$ of $a, b$ and $c$ respectively (so $\left[a^{\prime}, b^{\prime}\right],\left[b^{\prime}, c^{\prime}\right]$ and $\left[c^{\prime}, a^{\prime}\right]$ are the projections of the sides $[a, b],[b, c]$ and $[c, a]$ over $\gamma$ ). Thus, we have constructed a new triangle $T^{\prime}=\left\{a^{\prime}, b^{\prime}, c^{\prime}\right\}$ contained in the simple closed geodesic $\gamma$.

Next, we are going to construct some quadrilaterals from the sides of both triangles $T$ and $T^{\prime}$, in order to apply the previous lemmas.

As Figure 3 shows, every side of $T$ there is a simply connected locally geodesic quadrilateral:

(i) for $[a, b]$ we have $Q_{1}:=[a, b] \cup\left[a, a^{\prime}\right] \cup\left[b, b^{\prime}\right] \cup\left[a^{\prime}, b^{\prime}\right]$,

(ii) for $[b, c]$ we have $Q_{2}:=[b, c] \cup\left[b, b^{\prime}\right] \cup\left[c, c^{\prime}\right] \cup\left[b^{\prime}, c^{\prime}\right]$,

(iii) for $[c, a]$ we have $Q_{3}:=\left[c, a_{0}\right] \cup\left[c, c^{\prime}\right] \cup\left[a_{0}, a_{0}^{\prime}\right] \cup\left[c^{\prime}, a_{0}^{\prime}\right]$.

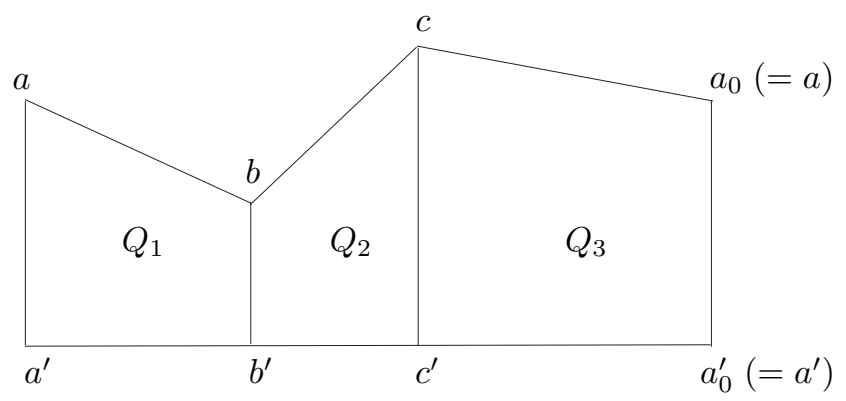

FIGURE 3

In the figure we have unfolded again our triangle in the universal covering surface to make things more understandable, and we have used two different names, $a$ and $a_{0}$, for the same vertex for the sake of simplicity in notation in the following argument. They are identified when the triangle is "glued" again (similarly for $a^{\prime}$ and $a_{0}^{\prime}$ of $\gamma$ ). Every $Q_{i}, 1 \leq i \leq 3$ is $2 \delta_{1}$-thin (its lift in the universal cover is a geodesic quadrilateral).

We claim that $T^{\prime}=\left\{a^{\prime}, b^{\prime}, c^{\prime}\right\}$ is a $\lambda$-triangle. Let us check it just for one side, for example $\left[a^{\prime}, b^{\prime}\right] \in Q_{1}$ :

(1) If $L_{S}\left(\left[a^{\prime}, b^{\prime}\right]\right)>\lambda / k$ then, by Lemma $3.5,\left[a, a^{\prime}\right] \cup\left[b, b^{\prime}\right] \cup\left[a^{\prime}, b^{\prime}\right]$ is a $(1, \lambda / k)$-quasigeodesic, so $\left[a^{\prime}, b^{\prime}\right] \subset \gamma$ is also a $(1, \lambda / k)$-quasigeodesic.

(2) If $L_{S}\left(\left[a^{\prime}, b^{\prime}\right]\right) \leq \lambda / k$ then, by Lemma 3.6 , the side $\left[a^{\prime}, b^{\prime}\right] \subset \gamma$ is a $(1, \lambda / k)$-quasigeodesic.

It follows that, on the one hand, $T^{\prime}$ is $\delta_{0}$-thin (by hypothesis) and, on the other hand, the lengths of at least two sides of $T^{\prime}$ are greater than or equal to $l / 4-\lambda /(2 k)$ (by Lemma 3.9). In our current case, as $l>10 \lambda / k$, it is easy to check that, actually, these lengths are greater than $2 \lambda / k$. Besides, note that: 


$$
\frac{l}{4}-\frac{\lambda}{2 k}>\frac{2 \lambda}{k}=\frac{2 \log (5+2 \sqrt{6})}{k}>\frac{2 \log 9}{k}=\frac{4 \log 3}{k}>\frac{4 \log (1+\sqrt{2})}{k}=4 \delta_{1} .
$$

This means that, at least two sides have length strictly greater than $4 \delta_{1}$.

Let $z$ be a point of one of the sides of $T$ and $\sigma$ the union of the other two sides; we are going to prove that $d(z, \sigma) \leq 12 \delta_{1}+\delta_{0}$. Without loss of generality we can assume that $z \in[a, b]$, and then there exists $z_{0} \in\left[a, a^{\prime}\right] \cup\left[b, b^{\prime}\right] \cup\left[a^{\prime}, b^{\prime}\right]$ with $d\left(z, z_{0}\right) \leq 2 \delta_{1}$, since $Q_{1}$ is $2 \delta_{1}$-thin.

If $z_{0} \in\left[a^{\prime}, b^{\prime}\right]$, since the triangle $T^{\prime}$ is $\delta_{0}$-thin, there exists $z_{0}^{\prime} \in\left[b^{\prime}, c^{\prime}\right] \cup\left[c^{\prime}, a_{0}^{\prime}\right]$ with $d\left(z_{0}, z_{0}^{\prime}\right) \leq \delta_{0}$. There is no loss of generality in assuming that $z_{0}^{\prime} \in\left[b^{\prime}, c^{\prime}\right]$. There are two possibilities:

(1) If $L_{S}\left(\left[b^{\prime}, c^{\prime}\right]\right)>4 \delta_{1}$, applying Lemma 3.7 we have that $d\left(z_{0}^{\prime},[b, c]\right) \leq 4 \delta_{1}$. Therefore $d(z, \sigma) \leq 6 \delta_{1}+\delta_{0}$.

(2) If $L_{S}\left(\left[b^{\prime}, c^{\prime}\right]\right) \leq 4 \delta_{1}$, then $d\left(z_{0}^{\prime}, c^{\prime}\right) \leq 4 \delta_{1}$. Note that $L_{S}\left(\left[c^{\prime}, a_{0}^{\prime}\right]\right)>4 \delta_{1}$ by (4.3) and by Lemma 3.7, $d\left(c^{\prime},\left[c, a_{0}\right]\right) \leq 4 \delta_{1}$. Therefore $d(z, \sigma) \leq 10 \delta_{1}+\delta_{0}$.

If $z_{0} \in\left[a, a^{\prime}\right] \cup\left[b, b^{\prime}\right]$, without loss of generality we can assume that $z_{0} \in\left[b, b^{\prime}\right] \in Q_{2}$. Since $Q_{2}$ is $2 \delta_{1}$-thin, once again, there exists $z_{0}^{\prime} \in[b, c] \cup\left[c, c^{\prime}\right] \cup\left[b^{\prime}, c^{\prime}\right]$ with $d\left(z_{0}, z_{0}^{\prime}\right) \leq 2 \delta_{1}$. If $z_{0}^{\prime} \in[b, c]$ then $d(z, \sigma) \leq 4 \delta_{1}$ and we are done. If $z_{0}^{\prime} \in\left[b^{\prime}, c^{\prime}\right] \cup\left[c, c^{\prime}\right]$, then there are two possibilities:

(1) If $L_{S}\left(\left[b^{\prime}, c^{\prime}\right]\right)>4 \delta_{1}$, then we can choose $z_{0}^{\prime}$ with $z_{0}^{\prime} \in\left[b^{\prime}, c^{\prime}\right]$ : this is because we can lift $Q_{2}$ to the universal covering $\tilde{S}$, obtaining in it a new geodesically convex quadrilateral (let us use the same notation for the points in $\tilde{S})$, hence $d_{\tilde{S}}\left(\left[b, b^{\prime}\right],\left[c, c^{\prime}\right]\right)=L_{\tilde{S}}\left(\left[b^{\prime}, c^{\prime}\right]\right)>4 \delta_{1}$. The new geodesic quadrilateral in $\tilde{S}$ is $2 \delta_{1}$-thin as well, so we can take a point $z_{0}^{\prime} \in\left[b^{\prime}, c^{\prime}\right] \subset \tilde{S}$ such that $d\left(z_{0}, z_{0}^{\prime}\right) \leq$ $d_{\tilde{S}}\left(z_{0}, z_{0}^{\prime}\right) \leq 2 \delta_{1}$. Applying now Lemma 3.7 we have that $d\left(z_{0}^{\prime},[b, c]\right) \leq 4 \delta_{1}$, and therefore $d(z, \sigma) \leq$ $8 \delta_{1}$.

(2) If $L_{S}\left(\left[b^{\prime}, c^{\prime}\right]\right) \leq 4 \delta_{1}$, there are two possible situations: either $z_{0}^{\prime} \in\left[b^{\prime}, c^{\prime}\right]$ or $z_{0}^{\prime} \in\left[c, c^{\prime}\right]$.

In the first case, $d_{S}\left(z_{0}^{\prime}, c^{\prime}\right) \leq 4 \delta_{1}$ and, since $L_{S}\left(\left[c^{\prime}, a_{0}^{\prime}\right]\right)>4 \delta_{1}$ (by (4.3)), applying Lemma 3.7 we have that $d\left(c^{\prime},\left[c, a_{0}\right]\right) \leq 4 \delta_{1}$; thus $d(z, \sigma) \leq 12 \delta_{1}$.

In the second case, when $z_{0}^{\prime} \in\left[c, c^{\prime}\right]$, notice that now we are in the quadrilateral $Q_{3}$ where $L\left[c^{\prime}, a_{0}^{\prime}\right]>$ $4 \delta_{1}$, and repeating the same arguments we obtain $d(z, \sigma) \leq 12 \delta_{1}$.

Hence, $S$ is $\delta$-thin, with $\delta:=\max \left\{18 \delta_{1}+20 \lambda / k, 12 \delta_{1}+\delta_{0}\right\}$.

One might think that the $\lambda$-triangle $T^{\prime}$ contained in the simple closed geodesic is geodesic; however, the following example shows that $T^{\prime}$ in the proof of Theorem 4.1 does not need to be geodesic, even in the constant curvature case.

Example. There is a geodesic triangle $T$ in a complete Riemannian surface $S$ with constant curvature $K=-k^{2}<0$, such that the corresponding $T^{\prime}$ is not geodesic. That is, at least one of the sides of $T^{\prime}$ is not geodesic (See Figure 4).

Given $x_{0}>0$ satisfying $k x_{0}<\operatorname{Arcsinh} 1$, let us fix $y>0$ with $\sinh k\left(x_{0}+y\right)>\cosh k y$. Then $\sinh k(x+y)>$ $\cosh k y$ for any $x$ satisfying $k x_{0} \leq k x<\operatorname{Arcsinh} 1$, and consequently we can choose $x>0$ such that $k x<\operatorname{Arcsinh} 1$ and $\sinh k x \sinh k(x+y)>\cosh k y$.

Let $\varepsilon:=\frac{1}{k} \operatorname{Arcsinh}(1 / \sinh k x)-x>0$, then $\sinh k x \sinh k(x+\varepsilon)=1$. Let us consider a geodesic quadrilateral $V$ in the Cartan-Hadamard surface $H_{k}$ with constant curvature $K=-k^{2}$, with three right angles and an angle equal to zero, such that the two finite sides have length $x$ and $x+\varepsilon$ (see e.g. [11, p.157] and [18, p.89]). If we glue together four isometric copies of $V$, we obtain a generalized $Y$-piece $Y$ with two punctures and a simple closed geodesic $\gamma$ with $L_{S}(\gamma)=4(x+\varepsilon)$. We obtain $S$ by gluing $Y$ with a funnel $F$ with $K=-k^{2}$ whose simple closed geodesic has length $4(x+\varepsilon)$.

Let us denote by $\mu_{0}$ the geodesic in $Y$ with $L_{S}\left(\mu_{0}\right)=2 x$, joining $\gamma$ with itself which is not homotopic to any curve contained in $\gamma$. We denote by $p^{\prime}, q^{\prime}$ the endpoints of $\mu_{0}$. Let us consider the unbounded geodesic $\mu$ in $S$ which contains $\mu_{0}$, and the two points $p, q \in \mu \cap F$ at distance $y$ of $\gamma$. 
Let us define the triangle $T$ as the union of the two geodesics $\alpha, \beta$ in $F$ joining $p$ and $q$ (in fact, $T$ is a geodesic "bigon"). The length of the segment of $\mu$ between $p$ and $q$ is $2 x+2 y$; by [18, p.89] we have $\sinh \left(k L_{S}(\alpha) / 2\right)=\sinh k(x+\varepsilon) \cosh k y=\cosh k y / \sinh k x<\sinh k(x+y)$. So we obtain $L_{S}(\alpha)<2 x+2 y=$ $L_{S}([p, q])$, and consequently $\alpha, \beta$ are in fact geodesics in $S$. However, $T^{\prime}=\left\{p^{\prime}, q^{\prime}\right\}$ is contained in $\gamma$ and $L_{S}\left(\alpha^{\prime}\right)=L_{S}\left(\beta^{\prime}\right)=2 x+2 \varepsilon>2 x=L_{S}\left(\mu_{0}\right)=d\left(p^{\prime}, q^{\prime}\right)$; hence $\alpha^{\prime}, \beta^{\prime}$ are not geodesics in $S$.

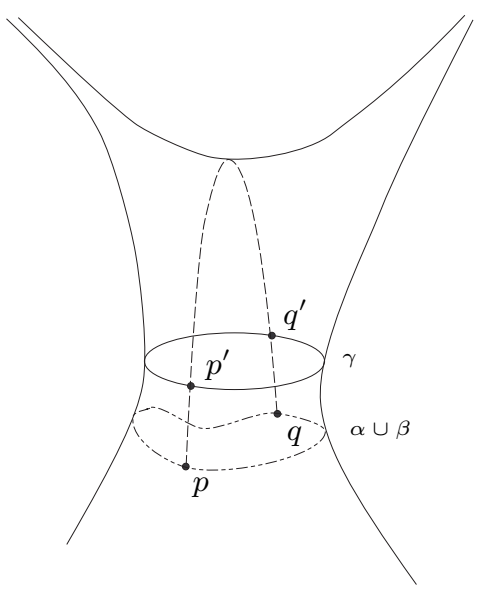

FiguRE 4

\section{Applications.}

In this section we will give several applications of Theorem 4.1.

Definition 5.1. We say that a complete Riemannian surface $S$ (with or without boundary) is of finite type if its fundamental group is finitely generated.

Corollary 5.2. Let us consider a complete Riemannian surface $S$ (with or without boundary) with $K \leq$ $-k^{2}<0$; if $S$ has boundary, we also require that $\partial S$ is the union of local geodesics (closed or non-closed). If $S$ is of finite type, then it is hyperbolic. More precisely, if $S$ is of finite type and every simple closed geodesic $\gamma$ in $S$ verifies $L_{S}(\gamma) \leq l$, then $S$ is $\delta$-thin, with $\delta=\max \left\{18 \delta_{1}+20 \lambda / k, 12 \delta_{1}+l / 4+\lambda /(2 k)\right\}$, where $\delta_{1}:=\frac{1}{k} \log (1+\sqrt{2})$ and $\lambda:=\log (5+2 \sqrt{6})$.

Proof. The number of simple closed geodesics in $S$ which are homotopic to a geodesic triangle is finite: $\left\{\gamma_{1}, \ldots, \gamma_{k}\right\}$, and hence we have $L_{S}\left(\gamma_{j}\right) \leq l$. Every continuous injective $(1, \lambda / k)$-quasigeodesic with its arclength parametrization $g \subset \gamma_{j}$ verifies $L_{S}(g) \leq l / 2+\lambda / k$ by Lemma 3.9; hence $d(z, \partial g) \leq l / 4+\lambda /(2 k)$ for every $z \in g$. Then the hypothesis of Theorem 4.1 is verified with $\delta_{0}:=l / 4+\lambda /(2 k)$. Hence $S$ is $\delta$-thin with $\delta=\max \left\{18 \delta_{1}+20 \lambda / k, 12 \delta_{1}+l / 4+\lambda /(2 k)\right\} \leq 18 \delta_{1}+20 \lambda / k+l / 4$.

A consequence of this corollary is the following result.

Corollary 5.3. Every generalized $Y$-piece $S$ with $L_{S}\left(\gamma_{i}\right) \leq l$, where $\gamma_{i}(i=1,2,3)$ are the simple closed geodesics in $\partial S$, is $\delta$-thin, with $\delta=\max \left\{18 \delta_{1}+20 \lambda / k, 12 \delta_{1}+l / 4+\lambda /(2 k)\right\}$, where $\delta_{1}:=\frac{1}{k} \log (1+\sqrt{2})$ and $\lambda:=\log (5+2 \sqrt{6})$. 
Remark 5.4. As usual, we view a puncture as a simple closed geodesic with length equal to zero.

It is clear that a funnel contains infinitely many halfplanes.

Two additional results can be deduced from Theorem 4.1. The first one (see Theorem 5.5 below) allows us to simplify the topology of a surface in order to study its hyperbolicity: it assures that deleting funnels and halfplanes does not change the hyperbolicity of a Riemannian surface.

If one thinks of possible conjectures about hyperbolicity, the following one might sound reasonable: "If a Riemannian surface has a sequence of funnels $\left\{F_{n}\right\}_{n}$ with $\lim _{n \rightarrow \infty} L_{S}\left(\partial F_{n}\right)=\infty$, then it is not hyperbolic". However, in [39] we prove that this reasonable result is false indeed, and besides it turns out to be an important tool in the proof is Theorem 5.5.

Theorem 5.5. Let us consider a complete Riemannian surface $S$ (with or without boundary); if $S$ has boundary, we also require that $\partial S$ is the union of local geodesics (closed or non-closed). Let us denote by $F$ the union of some pairwise disjoint funnels and halfplanes of $S$. Let $S_{0}$ be the bordered complete Riemannian surface obtained by deleting from $S$ the interior of $F$. Then $S$ is hyperbolic if and only if $S_{0}$ is hyperbolic.

More precisely, if $S$ is $\delta$-thin (respectively, $\delta$-hyperbolic) then $S_{0}$ is $\delta$-thin (respectively, $\delta$-hyperbolic); and if $S_{0}$ is $\delta^{\prime}$-hyperbolic, then $S$ is $\delta$-thin, with $\delta=\max \left\{18 \delta_{1}+20 \lambda / k, 12 \delta_{1}+4 \delta^{\prime}+2 H\left(\delta^{\prime}, 1, \lambda / k\right)\right\}$ where $\delta_{1}:=\frac{1}{k} \log (1+\sqrt{2}), \lambda:=\log (5+2 \sqrt{6})$ and $H$ is the constant in Theorem 2.9.

Remark 5.6. We want to emphasize that in Theorem 5.5 there is no hypothesis about the length of the boundary curves of the funnels (this is not the case in corollaries 5.2 and 5.3). This is an important fact since there are complete hyperbolic Riemannian surfaces containing funnels $F_{n}$ with $L_{S}\left(\partial F_{n}\right) \longrightarrow \infty$ as $n \rightarrow \infty$.

Proof. Let us assume that $S$ is $\delta$-thin (respectively, $\delta$-hyperbolic). As $S_{0}$ is geodesically convex in $S$ (every geodesic connecting two points of $S_{0}$ is contained in $\left.S_{0}\right)$, we have $d(z, w)=d_{S_{0}}(z, w)$ for every $z, w \in S_{0}$. Therefore $S_{0}$ is also $\delta$-thin (respectively, $\delta$-hyperbolic).

Let us assume now that $S_{0}$ is $\delta^{\prime}$-hyperbolic. By [39, Lemma 3.3] (or the first part of the proof of Theorem 4.1), every $\lambda$-triangle $T$ in $S_{0}$ is $\left(\delta^{\prime}+2 H\left(\delta^{\prime}, 1, \lambda / k\right)\right)$-thin, where $H$ is the constant in Theorem 2.9. Let us observe that any simple closed geodesic in $S$ is contained in $S_{0}$. Since $d(z, w)=d_{S_{0}}(z, w)$ for every $z, w \in S_{0}$, every $\lambda$-triangle in $S$ (contained in a simple closed geodesic in $S$ ) is also a $\lambda$-triangle in $S_{0}$. Let us observe also that $H \geq 1>\log (1+\sqrt{2})$. Then Theorem 4.1 implies that $S$ is $\delta$-thin, with $\delta=\max \left\{18 \delta_{1}+20 \lambda / k, 12 \delta_{1}+4 \delta^{\prime}+2 H\left(\delta^{\prime}, 1, \lambda / k\right)\right\}$.

Many Riemannian surfaces can be decomposed as a union of funnels and generalized $Y$-pieces (see [5] and [34, Theorem 4.1]). The following result uses this decomposition in order to obtain hyperbolicity.

Theorem 5.7. Let us consider a complete Riemannian surface $S$ with $-k_{1}^{2} \leq K \leq-k_{2}^{2}<0$ (with or without boundary), with genus equal to zero. If there is a decomposition of $S$ as a union of funnels $\left\{F_{m}\right\}_{m \in M}$ and generalized $Y$-pieces $\left\{Y_{n}\right\}_{n \in N}$ with $L_{S}(\gamma) \leq l$ for every simple closed geodesic $\gamma \subset\left(\cup_{n} \partial Y_{n}\right) \cup\left(\cup_{m} \partial F_{m}\right)$, then $S$ is $\delta$-hyperbolic, where $\delta$ is a constant depending only on $k_{1}, k_{2}$ and $l$.

Proof. The Collar Lemma in variable negative curvature (see [16]) states that there exists a constant $c_{0}$, which only depends on $k_{1}, k_{2}$ and $l$, such that $d\left(\gamma_{1}, \gamma_{2}\right) \geq c_{0}$ for every $\gamma_{1}, \gamma_{2} \subset\left(\cup_{n} \partial Y_{n}\right) \cup\left(\cup_{m} \partial F_{m}\right)$ with $\gamma_{1} \neq \gamma_{2}$.

Hence, $\left\{F_{m}, Y_{n}\right\}_{m, n}$ is a $\left(l / 2, c_{0}\right)$-tree decomposition of $S$.

By Lemma $3.10, F_{m}$ is $\tilde{\delta}$-hyperbolic for every $m \in M$, where $\tilde{\delta}$ is a constant which just depends on $k_{2}$ and $l$. By Corollary 5.3, $Y_{n}$ is $\delta^{*}$-hyperbolic for every $n \in N$, where $\delta^{*}$ is a constant which just depends on $k_{2}$ and $l$. Now the result follows from Theorem 2.14. 


\section{REFERENCES}

[1] Aikawa, H., Positive harmonic functions of finite order in a Denjoy type domain, Proc. Amer. Math. Soc. 131 (2003), 3873-3881.

[2] Anderson, J. W., Hyperbolic Geometry. Springer, London, 1999.

[3] Alvarez, V., Pestana, D., Rodríguez, J. M., Isoperimetric inequalities in Riemann surfaces of infinite type, Rev. Mat. Iberoamericana 15 (1999), 353-427.

[4] Alvarez, V., Portilla, A., Rodríguez, J. M., Tourís, E., Gromov hyperbolicity of Denjoy domains, Geom. Dedicata. In press.

[5] Alvarez, V., Rodríguez, J. M., Structure theorems for Riemann and topological surfaces, J. London Math. Soc. 69 (2004), 153-168.

[6] Alvarez, V., Rodríguez, J.M., Yakubovich, D.V., Subadditivity of p-harmonic "measure" on graphs, Michigan Math. J. 49 (2001), 47-64.

[7] Balogh, Z. M., Buckley, S. M., Geometric characterizations of Gromov hyperbolicity, Invent. Math. 153 (2003), $261-301$.

[8] Basmajian, A., Constructing pair of pants, Ann. Acad. Sci. Fenn. Series AI 15 (1990), 65-74.

[9] Basmajian, A., Hyperbolic structures for surfaces of infinite type, Trans. Amer. Math. Soc. 336 (1993), $421-444$

[10] Benoist, Y., Convexes hyperboliques et fonctions quasisymtriques, Publ. Math. Inst. Hautes Études Sci. 97 (2003), $181-237$.

[11] Bonk, M., Quasi-geodesics segments and Gromov hyperbolic spaces, Geom. Dedicata 62 (1996), 281-298.

[12] Bonk, M., Heinonen, J., Koskela, P., Uniformizing Gromov hyperbolic spaces. Astérisque No. 270 (2001).

[13] Buser, P., Geometry and Spectra of Compact Riemann Surfaces. Birkhäuser, Boston, 1992.

[14] Cantón, A., Fernández, J. L., Pestana, D., Rodríguez, J. M., On harmonic functions on trees, Potential Analysis 15 (2001), 199-244.

[15] Chavel, I., Eigenvalues in Riemannian Geometry. Academic Press, New York, 1984.

[16] Chavel, I., Feldman, E.A., Cylinders on surfaces, Comment. Math. Helvetici 53 (1978), 439-447.

[17] Eberlein, P., Surfaces of nonpositive curvature. Memoirs of the American Mathematical Society, Volume 20 , Number 218. Providence, Rhode Island. 1979.

[18] Fenchel, W., Elementary Geometry in Hyperbolic Space. Walter de Gruyter, Berlin-New York, 1989.

[19] Fernández, J. L., Rodríguez, J. M., Area growth and Green's function of Riemann surfaces, Arkiv för matematik 30 (1992), 83-92.

[20] Ghys, E., de la Harpe, P., Sur les Groupes Hyperboliques d'après Mikhael Gromov. Progress in Mathematics, Volume 83. Birkhäuser. 1990.

[21] Gromov, M., Hyperbolic groups, in "Essays in group theory". Edited by S. M. Gersten, M. S. R. I. Publ. 8. Springer, 1987, 75-263.

[22] Gromov, M. (with appendices by M. Katz, P. Pansu and S. Semmes), Metric Structures for Riemannian and NonRiemannnian Spaces. Progress in Mathematics, Vol. 152. Birkhäuser, 1999.

[23] Haas, A., Dirichlet points, Garnett points and infinite ends of hyperbolic surfaces I, Ann. Acad. Sci. Fenn. Series AI 21 (1996), 3-29.

[24] Hästö, P. A., Gromov hyperbolicity of the $j_{G}$ and $\widetilde{j}_{G}$ metrics, Proc. Amer. Math. Soc. In press.

[25] Holopainen, I., Soardi, P. M., p-harmonic functions on graphs and manifolds, Manuscripta Math. 94 (1997), 95-110.

[26] Kanai, M., Rough isometries and combinatorial approximations of geometries of non-compact Riemannian manifolds, $J$. Math. Soc. Japan 37 (1985), 391-413.

[27] Kanai, M., Rough isometries and the parabolicity of Riemannian manifolds, J. Math. Soc. Japan 38 (1986), $227-238$.

[28] Kanai, M., Analytic inequalities and rough isometries between non-compact Riemannian manifolds. Curvature and Topology of Riemannian manifolds (Katata, 1985). Lecture Notes in Math. 1201. Springer (1986), 122-137.

[29] Karlsson, A., Noskov, G. A., The Hilbert metric and Gromov hyperbolicity, Enseign. Math. 48 (2002), 73-89.

[30] Portilla, A., Rodríguez, J. M., Tourís, E., Gromov hyperbolicity through decomposition of metric spaces II, J. Geom. Anal. 14 (2004), 123-149.

[31] Portilla, A., Rodríguez, J. M., Tourís, E., The topology of balls and Gromov hyperbolicity of Riemann surfaces, Diff. Geom. Appl. 21 (2004), 317-335.

[32] Portilla, A., Rodríguez, J. M., Tourís, E., The role of funnels and punctures in the Gromov hyperbolicity of Riemann surfaces. Proc. Edinburgh Math. Soc. 49 (2006), 399-425.

[33] Portilla, A., Rodríguez, J. M., Tourís, E., A real variable characterization of Gromov hyperboicity of flute surfaces. Preprint.

[34] Portilla, A., Rodríguez, J. M., Tourís, E., Structure Theorem for Riemannian surfaces with arbitrary curvature. Preprint.

[35] Ratcliffe, J.G., Foundations of Hyperbolic Manifolds. Springer-Verlag, New York, 1994.

[36] Rodríguez, J. M., Isoperimetric inequalities and Dirichlet functions of Riemann surfaces, Publicacions Matemàtiques 38 (1994), 243-253.

[37] Rodríguez, J. M., Two remarks on Riemann surfaces, Publicacions Matemàtiques 38 (1994), 463-477.

[38] Rodríguez, J. M., Tourís, E., Gromov hyperbolicity through decomposition of metric spaces, Acta Math. Hung. 103 (2004), 53-84.

[39] Rodríguez, J. M., Tourís, E., A new characterization of Gromov hyperbolicity for negetively curved surfaces, Publicacions Matemàtiques 50 (2006), 249-278. 
[40] Rodríguez, J. M., Tourís, E., Gromov hyperbolicity of Riemann surfaces, Acta Math. Sinica. In press.

[41] Soardi, P. M., Rough isometries and Dirichlet finite harmonic functions on graphs, Proc. Amer. Math. Soc. 119 (1993), $1239-1248$.

[42] Väisälä, J., Hyperbolic and uniform domains in Banach spaces. Preprint.

[Available at http://www.helsinki.fi/juvaisala/preprints.html]

E-mail address: apferrei@math.uc3m.es, etouris@math.uc3m.es 